\title{
ÜBER DAS GEMISCHTE RANDWERTPROBLEM FÜR ELLIPTISCHE GLEICHUNGEN IN $n$-DIMENSIONALEN GEBIETEN MIT KANTEN
}

\author{
A. AZZAM und E. KREYSZIG
}

\section{Einleitung}

In der vorliegenden Arbeit untersuchen wir das gemischte Randwertproblem für gleichmässig elliptische Gleichungen

$$
L u=a_{i j}(x) u_{x_{i} x_{j}}+a_{i}(x) u_{x_{i}}+a(x) u=f(x)
$$

(Summationen gemäß Summationsvereinbarung), $x=\left(x_{1}, \ldots, x_{n}\right)$, mit $C^{\alpha}$-Koeffizienten in einem einfachzusammenhängenden beschränkten Gebiet $\Omega \subset \boldsymbol{R}^{n}, n \geqq 2$, dessen Rand $\partial \Omega$ Kanten besitzt. Die Randbedingungen sind dabei derart, dass jeweils $u=0$ bzw. $u_{v}=\partial u / \partial v=0$ auf zwei Hyperflächenstücken $\Gamma_{1}$ bzw. $\Gamma_{2}$ gilt, deren Schnitt eine $(n-2)$-dimensionale Kante bildet (genaue Voraussetzungen s. unten). Hierbei ist $v$ die äussere Normale.

Die konforme Abbildung hat erstmals Fragen gezeitigt, die auf spezielle derartige Randwertprobleme führten. Später wurden Sätze über das kantennahe Lösungsverhalten beim allgemeinen Problem grundlegend für die Theorie der Differenzenverfahren (vgl. P. Laasonen [5], [6]) und für die Methode der finiten Elemente. Während die meisten bisherigen Arbeiten das Dirichletproblem betreffen (s. die in [3] genannte Literatur), betrachten wir hier die gemischte Randwertaufgabe und beweisen in Abschnitt 3 Glattheitsaussagen für Lösungen bis zum Rand von Gebieten der obigen Art. Letztere folgen aus Ergebnissen im Sonderfalle eines Zylindersektors, der nun behandelt wird.

\section{Das Problem für einen Zylindersektor}

Betrachtet wird

$$
L_{0} U=A_{i j}(y) U_{y_{i} y_{j}}+A_{i}(y) U_{y_{i}}+A(y) U=F(y),
$$

$y=\left(y_{1}, \ldots, y_{n}\right)$, in einem Zylindersektor

$$
\begin{aligned}
& G_{c}=\left\{\left(\varrho, \theta, y^{\prime}\right)\left|\theta_{1}<\theta<\theta_{2},\right| y \mid<c\right\}, \\
& \theta_{1}=\frac{1}{2} \pi-\omega, \quad \theta_{2}=\frac{1}{2} \pi, \quad \omega<\frac{1}{2} \pi .
\end{aligned}
$$


Hierbei ist

$$
\begin{aligned}
y_{1} & =\varrho \cos \theta, \quad y_{2}=\varrho \sin \theta \\
y^{\prime} & =\left(y_{3}, \ldots, y_{n}\right) \quad \text { falls } \quad n \geqq 3 \\
|y|^{2} & =y_{1}^{2}+\ldots+y_{n}^{2} .
\end{aligned}
$$

Wir machen die folgenden Voraussetzungen:

(V1) $L_{0}$ gleichmässig elliptisch in $G_{c}$,

$$
A_{i j}, A_{i}, A, F \in C^{\alpha}\left(\bar{G}_{c}\right), \quad 0<\alpha<1 .
$$

(V2) $A_{i j}(0)=\delta_{i j}$ für $i, j=1,2$.

(V3) $\left.U\right|_{\Gamma_{c}^{(1)}}=0,\left.U_{v}\right|_{\Gamma_{c}^{(2)}}=0$,

Dann gilt der

$$
\Gamma_{c}^{(j)}=\left\{y \mid y \in \partial G_{c}, \theta=\theta_{j}\right\}, \quad j=1,2 .
$$

Satz 2.1. Es sei $U$ eine beschränkte Lösung von (2.1) in $G_{c}$ mit $c<1$, und (V1)(V3) seien erfüllt. Dann existiert ein positives $r_{0}<c / 3$ derart, dass gilt

$$
U \in C^{\mu}\left(\bar{G}_{r_{0}}\right)
$$

mit

mit beliebig kleinem $\varepsilon>0$.

$$
\mu=\left\{\begin{array}{ccc}
2 & \text { für } & \pi / 2 \omega>2 \\
\pi / 2 \omega-\varepsilon & \text { für } & \pi / 2 \omega \leqq 2
\end{array}\right.
$$

Dieser Satz ergibt sich aus dem

Lemma 2.2. Unter den Voraussetzungen des Satzes 2.1 ist in $G_{3 \tilde{r}_{0}}$

$$
|U(y)| \leqq \tilde{M} \varrho^{\mu}
$$

mit $\tilde{M}>0$ und $\mu$ gemäss (2.3).

Beweis. Wegen (V1) ist $F$ beschränkt, etwa $|F(y)| \leqq R$ in $G_{c}$. Ohne Beschränkung der Allgemeinheit können wir $\left.U\right|_{B}=0$ mit $B \subset \partial G_{3 r_{0}}$ gegeben durch $|y|=3 r_{0}$ annehmen; vgl. [1]. Es sei

$$
V(y)=M \varrho^{\mu} \cos \lambda\left(\frac{1}{2} \pi-\theta\right)
$$

mit $y=\left(\varrho, \theta, y^{\prime}\right) \in G_{3 r_{0}}, \mu$ gemäß (2.3) und

$$
\lambda=(\pi-2 \delta) / 2 \omega>\mu .
$$

$M>0$ und $r_{0}>0$ werden später geeignet gewählt. Wir setzen

$$
\begin{aligned}
& L_{0} V=L_{1} V+L_{2} V, \\
& L_{1} V=\Delta V+\sum_{i, j=1}^{2}\left(A_{i j}-\delta_{i j}\right) V_{y_{i} y_{j}} .
\end{aligned}
$$


Hierbei ist $\Delta=\partial^{2} / \partial y_{1}^{2}+\partial^{2} / \partial y_{2}^{2}$, und $A_{i j}(y)-\delta_{i j}$ ist stetig und verschwindet für $y=0$. Da für $\theta_{1} \leqq \theta \leqq \theta_{2}$

$$
\sin \delta \leqq \cos \lambda\left(\frac{1}{2} \pi-\theta\right) \leqq 1
$$

gilt, hat man die Abschätzung

$$
L_{1} V(y) \leqq-M\left[\left(\lambda^{2}-\mu^{2}\right) \sin \delta-\varepsilon_{1}\right] \varrho^{\mu-2}
$$

in $G_{3 r_{0}}$ mit hinreichend kleinem $r_{0}>0$ und beliebig kleinem $\varepsilon_{1}>0$. Nun enthält $L_{2} V$ höchstens erste Ableitungen nach $y_{1}$ und $y_{2}$. Also ist

$$
L_{2} V(y)=o\left(\varrho^{\mu-2}\right) .
$$

Wählen wir $\varepsilon_{1}<\left(\lambda^{2}-\mu^{2}\right) \sin \delta$ und dann $r_{0}$ hinreichend klein sowie $M$ hinreichend gross, so folgt

also

$$
L_{0} V(y) \leqq-R \quad \text { in } \quad G_{3 r_{0}},
$$

$$
L_{0} V(y) \leqq F(y) \text { in } G_{3 r_{0}} .
$$

Wir schätzen $V$ bzw. $V_{v}$ auf $\partial G_{3 r_{0}}$ ab. Es gilt für $\theta=\theta_{1}$

$$
V(y)=M \varrho^{\mu} \sin \delta \geqq 0,
$$

auf $B$

und für $\theta=\theta_{2}$

$$
\begin{aligned}
V(y) & =M\left(3 r_{0}\right)^{\mu} \cos \lambda\left(\frac{1}{2} \pi-\theta\right) \\
& \geqq M\left(3 r_{0}\right)^{\mu} \sin \delta>0
\end{aligned}
$$

Entsprechend ergibt sich für

$$
V_{v}=\frac{1}{\varrho} V_{\theta}=0 .
$$

$$
W(y)=-M^{*} \varrho^{\mu} \cos \lambda\left(\frac{1}{2} \pi-\theta\right)
$$

mit hinreichend grossem $M^{*}$ bei hinreichend kleinem $r_{0}^{*}$

und auch auf $B$ sowie

$$
\begin{aligned}
L_{0} W(y) & \geqq F(y) \text { in } \quad G_{3 r_{0}} \\
W(y) & \leqq 0 \quad \text { für } \quad \theta=\theta_{1}
\end{aligned}
$$

$$
W_{v}(y)=0 \text { für } \theta=\theta_{2} .
$$

Aus dem Maximumprinzip (s. C. Miranda [7]) folgt nun in $\bar{G}_{3 \tilde{r}_{0}}$, wobei $\tilde{r}_{0}=$ $\min \left(r_{0}, r_{0}^{*}\right)$ bezeichnet,

$$
W(y) \leqq U(y) \leqq V(y) .
$$

Indem wir $\tilde{M}=\max \left(M, M^{*}\right)$ setzen, ergibt sich nun (2.4).

Wie man sieht, genügt es, in diesem Beweis statt (V1) nur

$\left(\mathrm{V} 1^{*}\right) L_{0}$ gleichmässig elliptisch in $G_{c}$,

$A_{i j}$ stetig in $\bar{G}_{c}$,

$A_{i}, A, F$ beschränkt in $\bar{G}_{c}$

vorauszusetzen. 
Aus Lemma 2.2 folgt nun Satz 2.1 ähnlich wie in [2], nämlich, indem man vermöge einer Schauder-Ungleichung Abschätzungen für die Ableitungen gewinnt, aus denen sich dann Satz 2.1 ergibt.

\section{Das Problem für ein allgemeines Gebiet mit Kanten}

Für ein gemischtes Randwertproblem

$$
\begin{gathered}
L u=a_{i j}(x) u_{x_{i} x_{j}}+a_{i}(x) u_{x_{i}}+a(x) u=f(x) \text { in } \Omega \\
\psi_{1}(x) u(x)+\psi_{2}(x) u_{v}(x)=\varphi(x) \text { auf } \partial \Omega
\end{gathered}
$$

( $L$ gleichmässig elliptisch in $\Omega, \psi_{1}^{2}+\psi_{2}^{2} \neq 0, u_{v}=\partial u / \partial v$ die Ableitung in Richtung der äusseren Normalen) gilt bekanntlich: Ist $\partial \Omega$ glatt, so verbessert sich die Glattheit der Lösung bis zum Rand mit wachsender Glattheit der Koeffizienten und Randdaten.

Für Gebiete $\Omega$ mit nichtglattem Rand gilt Vorstehendes aber nicht mehr. Einem solchen $\Omega \subset \boldsymbol{R}^{n}$ mit (n-2)-dimensionalen Kanten wenden wir uns nun zu. Da es sich um lokales Verhalten handelt, genügt es anzunehmen, $\partial \Omega=\Gamma_{1} \cup \Gamma_{2}$ bestehe aus zwei Hyperflächenstücken $\Gamma_{1}, \Gamma_{2}$ der Klasse $C^{2+\alpha}$, deren Schnitt $S=\Gamma_{1} \cap \Gamma_{2}$ eine Kante bildet. Wir betrachten also das Problem

$$
L u=f \quad \text { in } \Omega \text {, mit } L \text { gemäss (3.1) }
$$

$$
\begin{gathered}
\left.u\right|_{\Gamma_{1}}=0 \\
\left.u_{v}\right|_{\Gamma_{2}}=0 .
\end{gathered}
$$

Es gilt (vgl. [1]): Ist $a_{i j}, a_{i}, a, f \in C^{\alpha}(\bar{\Omega}), 0<\alpha<1$, so ist für die Lösung $u$ von (3.2) und (3.3)

$$
u \in C^{2+\alpha}\left(\Omega_{1}\right) \cap C^{0}(\bar{\Omega})
$$

in jedem beliebigen kompakten Bereich $\Omega_{1} \subset \bar{\Omega}$ mit positivem Abstand von $S$.

Um die Glattheit von $u$ in einer Umgebung von $S$ zu untersuchen, wählen wir einen beliebigen Punkt $P: x=x^{0}$ in $S$ und definieren

$$
\omega(P)=\arctan \frac{\left[a_{11}\left(x^{0}\right) a_{22}\left(x^{0}\right)-a_{12}^{2}\left(x^{0}\right)\right]^{1 / 2}}{a_{22}\left(x^{0}\right) \cot \gamma(P)-a_{12}\left(x^{0}\right)}
$$

wobei $\gamma(P)$ der Winkel zwischen $\Gamma_{1}$ und $\Gamma_{2}$ im Punkte $P$ sei. Offenbar erhält man $\omega(P)$ aus $\gamma(P)$ bei der Transformation von

auf Normalform. Wir setzen

$$
a_{i j}\left(x^{0}\right) u_{x_{i} x_{j}}=0
$$

$$
\omega=\max _{P \in S} \omega(P)
$$

mit $\omega(P)$ gemäss (3.5). Dann lässt sich das Hauptergebnis der Arbeit so fassen: 
Satz 3.1. Es sei u eine beschränkte Lösung des gemischten Problems (3.2), (3.3) in einem Gebiet $\Omega \subset \boldsymbol{R}^{n}$ der vorstehend genannten Art mit $\omega<\frac{1}{2} \pi$ in (3.7). Weiter sei L gleichmässig elliptisch in $\Omega$ und $a_{i j}, a_{i}, a, f \in C^{\alpha}(\bar{\Omega}), 0<\alpha<1$. Dann gilt

$$
u \in C^{\mu}(\bar{\Omega}) \text {, }
$$

mit beliebig kleinem $\varepsilon>0$.

$$
\mu=\left\{\begin{array}{ccc}
2 & \text { für } & \omega<\frac{1}{4} \pi \\
\pi / 2 \omega-\varepsilon & \text { für } & \omega \geqq \frac{1}{4} \pi
\end{array}\right.
$$

Beweis. Aus (3.4) sieht man, dass es genügt, für einen beliebigen Punkt $P \in S$

$$
u \in C^{\mu}\left(\bar{\Omega}_{P, b}\right)
$$

zu zeigen, wobei $\Omega_{P, b}$ der Schnitt von $\Omega$ mit einer offenen Kugel vom Radius $b$ mit dem Mittelpunkt $P$ ist. Es sei $P: x^{0}=\left(x_{1}^{0}, \ldots, x_{n}^{0}\right)$, und

$$
\begin{aligned}
& x_{1}=g\left(x_{2}, \ldots, x_{n}\right) \\
& x_{2}=h\left(x_{1}, x_{3}, \ldots, x_{n}\right)
\end{aligned}
$$

seien lokale Darstellungen von $\Gamma_{1}$ bzw. $\Gamma_{2}$ in einer Umgebung von $P$ mit $g$ und $h$ von der Klasse $C^{2+\alpha}$. Dann existiert eine Transformation der Form

$$
\begin{gathered}
y_{i}=\beta_{i 1}\left(x_{1}-g\right)+\beta_{i 2}\left(x_{2}-h\right)+\sum_{j=3}^{n} \beta_{i j}\left(x_{j}-x_{j}^{0}\right), \\
\beta_{i j} \text { konstant }, \quad i=1, \ldots, n,
\end{gathered}
$$

derart, dass gilt: (3.2) geht über in die elliptische Gleichung

$$
A_{i j}(y) U_{y_{i} y_{j}}+A_{i}(y) U_{y_{i}}+A(y) U=F(y)
$$

für $U(y)=u(x)$ mit $A_{i j}(0)=\delta_{i j}$ im Nullpunkt 0 , dem Bild von $P$, und in $P$ nichtverschwindender Jacobi-Determinante. Die Formel (3.9) geht über in die Darstellung zweier Hyperebenenstücke

$$
y_{1}=0, \quad y_{1}=y_{2} \tan \omega(P),
$$

die den Winkel $\omega(P)<\frac{1}{2} \pi$ bilden.

Diese Transformation (3.10) bildet ein Gebiet $\Omega_{P, b_{1}}$ der eingangs genannten Art auf ein Gebiet $G_{0,2 c}$ ab, das von (3.12) und einem Hyperflächenstück mit positivem Abstand $2 c$ von 0 berandet wird. In

$$
G_{c}=\left\{\left(\varrho, \theta, y^{\prime}\right)\left|\theta_{1}<\theta<\theta_{2},\right| y \mid<c\right\} \subset G_{0,2 c}
$$

erfüllt $U$ alle Bedingungen des Satzes 2.1 mit $\omega(P)$ statt $\omega$. Also ist $U \in C^{\mu(P)}\left(\bar{G}_{r_{0}}\right)$ mit $r_{0}<c / 3$ und

$$
\mu(P)=\left\{\begin{array}{ccc}
2 & \text { für } & \omega(P)<\frac{1}{4} \pi \\
\pi / 2 \omega(P)-\varepsilon & \text { für } & \omega(P) \geqq \frac{1}{4} \pi .
\end{array}\right.
$$


$\mathrm{Da}$ (3.10) von der Klasse $C^{2+\alpha}$ ist und die Jacobi-Determinante in $P$ nicht verschwindet, folgt

$$
u(x) \in C^{\mu(P)}\left(\bar{\Omega}_{P, b}\right) \quad\left(b<b_{1}\right),
$$

und $\Omega_{P, b}$ liegt im Urbild von $\bar{G}_{r_{0}}$. Der Satz ist damit bewiesen.

Bemerkung zu Satz 3.1. Für $\omega>\frac{1}{4} \pi$ brauchen die zweiten partiellen Ableitungen von $u$ nicht mehr in $\bar{\Omega}$ stetig zu sein, aber (ähnlich wie in [2]) kann man dann die Existenz von $\tau, \tau_{1} \in(0,1)$ zeigen, so dass

$$
r^{\tau} u_{x_{i} x_{j}} \in C^{\tau_{1}}(\bar{\Omega})
$$

gilt; $r$ ist dabei der Abstand von $S$ vom Punkte $x$, in dem der Wert der Ableitung genommen wird.

Wir danken N.S.E.R.C. für Unterstützung durch Grant A9097.

\section{Literatur}

[1] Agmon, S., A. Douglis, und L. Nirenberg: Estimates near the boundary for solutions of elliptic partial differential equations satisfying general boundary conditions. - Comm. Pure Appl. Math. 12, 1959, 623-727.

[2] Azzam, A.: On Dirichlet's problem for elliptic equations in sectionally smooth $n$-dimensional domains. - SIAM J. Math. Anal. 11, 1980, 248-253.

[3] Grisvard, P.: Behavior of the solutions of an elliptic boundary value problem in a polygonal or polyhedral domain, Numerical solutions of partial differential equations III, SYNSPADE 1975. - Academic Press, New York, 1976.

[4] LaAsonen, P.: On the behavior of the solution of the Dirichlet problem at analytic corners. Ann. Acad. Sci. Fenn. Ser. A I 241, 1957, 1-13.

[5] LaAsonen, P.: On the degree of convergence of discrete approximations for the solutions of the Dirichlet problem. - Ann. Acad. Sci. Fenn. Ser. A I 246, 1957, 1-19.

[6] Laasonen, P.: On the solution of Poisson's difference equation. - J. Assoc. Comput. Mach. $5,1958,370-382$.

[7] Miranda, C.: Partial differential equations of elliptic type. 2nd ed. - Ergebnisse der Mathematik und ihrer Grenzgebiete, Band 2. - Springer-Verlag, Berlin-Heidelberg-New York, 1970.

[8] Wasow, W.: Asymptotic development of the solution of Dirichlet's problem at analytic corners.

- Duke Math. J. 24, 1957, 47-56.

University of Windsor

Department of Mathematics

Windsor, Ontario

Canada

Eingegangen am 26. November 1979 